\title{
Nas encruzilhadas da bioquímica em Portugal:
}

\author{
a vida e a obra de Ruy Eugénio de Carvalho Pinto (1924-)
}

I S A B E L A M A R A L * E FER A N D O A N T U N E S**

Ruy Eugénio de Carvalho Pinto é um dos poucos discípulos de Kurt Jacobsohn que deu continuidade ao programa de investigação por ele iniciado em 1929, no Instituto de Investigação Científica Bento da Rocha Cabral (IRC). Realizou o estágio de licenciatura sob sua orientação no Departamento de Química da Faculdade de Ciências da Universidade de Lisboa e adquiriu formação especializada nas Universidades de Sheffield e Oxford, em Inglaterra. Regressado definitivamente a Portugal, em 1970, empenhou-se na criação da licenciatura em Bioquímica na Faculdade de Ciências de Lisboa, na década de 80. Desde então, tem congregado em seu redor vários se- guidores no âmbito da bioquímica experimental e teórica. Na tradição herdada de Ferreira de Mira, Ruy E. Pinto tem sido o mentor das tertúlias do Instituto Rocha Cabral (IRC), instituição que dirige desde 1996. Desde esta data, o IRC tem também acolhido vários grupos de investigação em História da Ciência, com os quais Ruy E. Pinto tem desenvolvido alguns projectos de investigação. Homenagear Ruy E. Pinto é homenagear um dos impulsionadores da bioquímica em Portugal, bem como todos aqueles que contribuíram para a consolidação e a afirmação desta disciplina científica, nascida na Alemanha, nas primeiras décadas do século passado.

\section{Breves Elementos Biográficos}

Ruy Eugénio Marques da Cunha Moreira Carvalho Pinto nasceu em Lisboa a 1 de Agosto de 1924. Seu pai, João Baptista Paes Moreira de Carvalho Pinto, era director de uma repartição na Junta do Crédito Público e, simultaneamente, professor na Escola Comercial Veiga Beirão, em Lisboa. Sua mãe, Filipina Ferreira Marques da Cunha Pinto, era doméstica.

Ruy Pinto nunca frequentou a instrução primária nem os dois primeiros anos do ensino liceal, pois teve o pai como único professor. Frequentou o Liceu Pedro Nunes onde fez o ensino secundário que concluiu em 1942.

\footnotetext{
* Centro de Investigação em História e Filosofia da Ciência e da Tecnologia, Faculdade de Ciências e Tecnologia/ Universidade Nova de Lisboa, Monte da Caparica, 2829-516 Caparica / Portugal; imsp-amaral@fct.unl.pt

** Departamento de Química e Bioquímica e Grupo de Bioquímica dos Oxidantes e Antioxidantes, Centro de Química e Bioquímica, da Faculdade de Ciências da Universidade de Lisboa, P-1749-016 Lisboa, Portugal; Instituto de Investigação Científica Bento da Rocha Cabral, P-1250047 Lisboa, Portugal; fantunes@fc.ul.pt
}

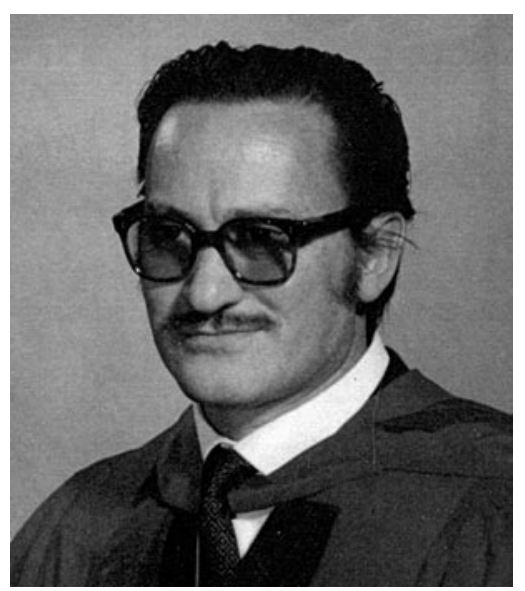

Figura 1 Fotografia de Ruy E. Pinto datada de 1970

(Fotografia gentilmente cedida pelo Professor Ruy E. Pinto)

A passagem do liceu para a faculdade revela uma das facetas da sua personalidade, que iria marcar toda a sua vida - a pluralidade de interesses que sempre o caracterizou [1]. Frequentou dois anos do curso de Belas Artes e o primeiro ano do curso preparatório de Engenharia, na Faculdade de Ciências da Universidade de Lisboa (FCUL). Con- correu, no ano seguinte, ao IST onde se manteve três anos. Regressou à Faculdade de Ciências em 1950 e, em 1953, concluiu $03 .^{\circ}$ ano do curso de Engenharia e a Licenciatura em Ciências Físico-Químicas. O estágio foi efectuado na FCUL e no IRC sob orientação de Kurt Jacobsohn, tendo por tema, Estudo da Urease em vários Tipos de Feijão.

Terminada a licenciatura, Ruy E. Pinto candidatou-se a um curso de bioquímica organizado por Hans Krebs, director do departamento de Bioquímica na Universidade de Oxford. Este curso, organizado anualmente, admitia seis doutorados ou licenciados de qualquer país, que possuíssem formação em Medicina, Biologia ou Química. O curso era leccionado pelos docentes e investigadores estrangeiros daquele departamento em colaboração com os departamentos de microbiologia, química orgânica e química inorgânica, da mesma universidade. Por intercessão de Jacobsohn, H. Krebs aceitou Ruy E. Pinto, o único candidato à frequência do curso que ainda não possuía doutoramento, nem era médico. Entre 1956 e 1957, frequentou aquela pós-gradu- 
ação em bioquímica na Universidade de Oxford, financiada pela Fundação Calouste Gulbenkian. Neste curso de especialização, sobre "conceitos e técnicas de bioquímica moderna,"[2] teve também como colega, Manuel Sobrinho Simões, na época primeiro assistente na Faculdade de Medicina do Porto. Ambos se decidiram por uma especialização em bioquímica num dos laboratórios de investigação com maior renome internacional, na época.

Ruy E. Pinto aventurou-se decididamente pela investigação bioquímica, não obstante algumas dificuldades que teve de gerir, em particular, de carácter financeiro. Tal como H. Krebs refere, em carta dirigida à Fundação Calouste Gulbenkian, Ruy E. Pinto era um bioquímico promissor que deveria dar continuidade à sua determinação e vontade de seguir uma carreira de investigação nesta área [2].

Tanto para Ruy Pinto como para Sobrinho Simões esta experiência em Oxford constituiu um desafio pessoal de extrema importância. No entanto, naquela época, a bioquímica em Portugal estava ainda em estado embrionário. Em Lisboa, apenas Kurt Jacobsohn se dedicava à investigação enzimológica de acordo com o programa de investigação da sua escola de investigação fundada em 1929; no Porto, Francisco Carvalho Guerra dava os primeiros passos para criar um grupo de investigação na Faculdade de Farmácia; e, em Coimbra, Arsélio Pato de Carvalho seguia-Ihe o exemplo, no Departamento de Zoologia da Faculdade de Ciências e Tecnologia. De facto, quando regressaram, tanto Ruy E. Pinto como Manuel Sobrinho Simões tiveram dificuldades em obter condições que Ihes permitissem desenvolver a investigação de forma regular, consistente e de acordo com os padrões científicos internacionais, nos quais tinham sido formados.

Em 1958, Ruy E. Pinto regressou a Portugal e, no ano seguinte, foi admitido como segundo assistente na FCUL, leccionando disciplinas de vários cursos. Em 1966, obteve o grau de doutor em química pela Universidade de Lisboa, fruto da investigação iniciada na Universidade de Oxford, com o título Con- tribuição para o Estudo da Glutationa. Oxidação-Redução em Homogenatos Hepáticos [3].

Em 1967, partiu novamente para Inglaterra como bolseiro do Instituto para a Alta Cultura e efectuou um curso de pós-doutoramento com a duração de 3 anos no Departamento de Bioquímica da Universidade de Sheffield, dirigido por Walter Bartley. Aproveitou parte deste trabalho de investigação para apresentar uma dissertação intitulada Studies of the Changes in the Activities of Glutathione Reductase and Glutathione Peroxidase and of the Aerobic Oxidation of Reduced Glutathione by Liver [3], que Ihe conferiu o grau de Doutor em Bioquímica na Universidade de Sheffield.

Embora pudesse ter optado por dar continuidade à carreira iniciada em Inglaterra ou noutros países, como o Canadá ou os Estados Unidos, Ruy E. Pinto decidiu regressar a Portugal. Entre 1970 e 1976, dirigiu o grupo de investigação em bioquímica no Laboratório de Física e Engenharia Nuclear (LFEN), na qualidade de director científico do Laboratório de Investigação em Biologia. Nos três anos seguintes, foi director do Departamento de Biologia do Laboratório Nacional de Engenharia e Tecnologia Industrial (LNETI).

Após a Jubilação de Kurt Jacobsohn, Ruy E. Pinto tornou-se professor convidado da FCUL e, em 1979, professor catedrático, com vínculo definitivo, a partir de 1981. É a ele que se deve a institucionalização da bioquímica na Faculdade de Ciências através da criação da respectiva licenciatura. Foi seu coordenador entre 1982 e 1994, altura em que se jubilou.

Ruy E. Pinto assumiu a direcção do IRC em 1996, uma instituição que o marcou pelo papel decisivo que tivera no início do século XX, no desenvolvimento da investigação experimental das Ciências Biológicas em Portugal. Ruy E. Pinto sucede, assim, a uma plêiade de directores que tanto marcaram a vida do IRC e o panorama científico nacional: Ferreira de Mira, Joaquim Fontes, Mirabeau Cruz e Kurt Jacobsohn. Foi, certamente, a consciência desta herança que o levou a apoiar a investigação histórica no seio do Instituto, a partir de 2001. Neste contexto, Ruy E. Pinto tem mantido acesa a animação das tertúlias anuais sobre variadíssimos temas, algumas delas dedicadas às figuras mais emblemáticas da instituição como sejam as palestras Ferreira de Mira e as conferências Kurt Jacobsohn.

Para além das actividades docentes e de investigação, Ruy E. Pinto acumulou outros cargos desde 1962. Foi consultor bioquímico do Hospital do Ultramar e do Hospital de Egas Moniz, foi membro do Conselho Médico-Legal, do Conselho Consultivo do Instituto Nacional de Investigação e Pescas, do Conselho Científico da FCUL, presidente do Conselho Pedagógico da FCUL, presidente da Assembleia de Representantes da FCUL, vogal da direcção do Departamento de Química e Bioquímica da FCUL, director do Centro de Estudos em Bioquímica e Fisiologia Animal da Universidade de Lisboa, e coordenador do Grupo de Bioquímica e Biologia Teóricas do IRC. Para além disso, foi ainda colaborador e consultor de algumas publicações nacionais: colaborador da Enciclopédia Verbo, consultor especializado da Enciclopédia Alfa, colaborador científico da Grande Enciclopédia Luso-Brasileira e membro do conselho científico da Revista Portuguesa de Bioquímica Aplicada e da Bioquímica Aplicada. Foi supervisor ou co-supervisor de mais de uma dezena de dissertações de doutoramento.

Ruy E. Pinto é autor de cerca de 200 títulos, que incluem artigos de divulgação científica e de carácter pedagógico, resumos apresentados em congressos científicos e artigos de investigação científica [3]. Apesar do número de trabalhos publicados não ser muito extenso, a obra científica de Ruy E. Pinto constituiu uma referência internacional no âmbito do metabolismo oxidativo, que a partir de 1961 passou a ser valorizada, como consequência do trabalho desenvolvido em Oxford e em Sheffield.

\section{A investigação bioquímica - o percurso de Ruy E. Pinto}

Desde a descoberta do glutationo por Hopkins em 1922, houve um grande interesse pelos estudos de oxidação-re- 


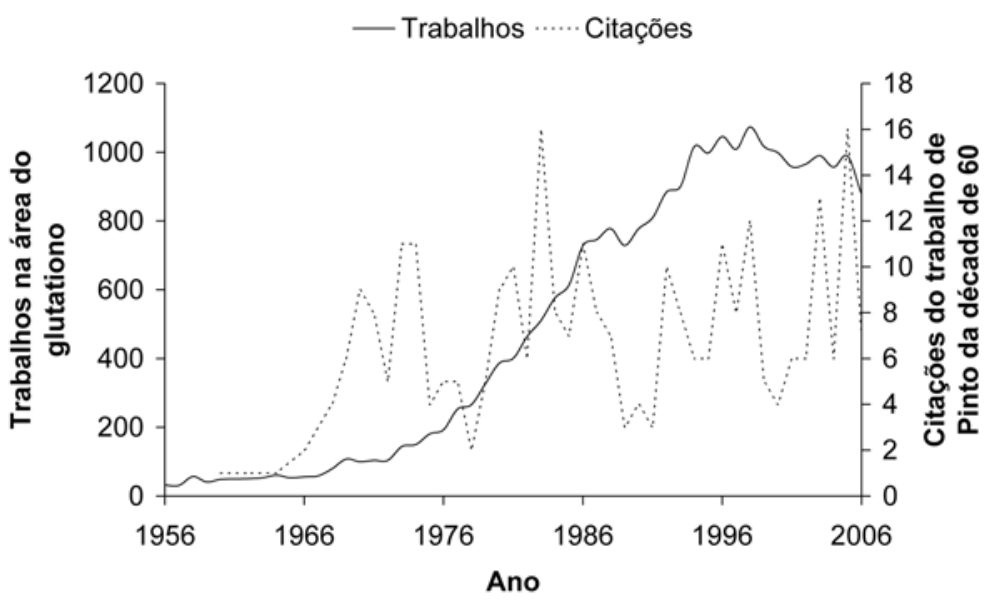

Figura 2 O trabalho de Ruy E. Pinto e o desenvolvimento da área da bioquímica do glutationo até $2006^{2}$

dução deste composto. Este entusiasmo viria a reduzir-se depois deste autor ter proposto, em 1931, que a oxidação do glutationo pelo fígado não era de natureza enzimática, nem catalisada por heminas.

Em 1961, Ruy Pinto mostrou existir oxidação enzimática do glutationo em meio aeróbio [4], facto que foi verificado pouco tempo depois por Jocelyn, em 1962 [5], e que constituiu um grande avanço no estudo metabólico do glutationo. A natureza enzimática da oxidação do glutationo foi estudada por este autor na Grã-Bretanha [6] e por Christopherson, na Noruega [7], e permitiu concluir que a oxidação do glutationo envolveria ainda os peróxidos lipídicos [8], para além da xantina-oxidase, como propôs Jocelyn [7].

A partir de 1967, Ruy E. Pinto começou a estudar, em Sheffield, a natureza da oxidação do glutationo que tinha descoberto. Em 1968 e 1969 mostrou que, muito provavelmente, a oxidação era essencialmente feita pelos peróxidos (pe- róxido de hidrogénio e peróxidos orgânicos), sendo a catálise efectuada pelo glutationo peroxidase. Estudou vários factores que afectam a actividade do glutationo peroxidase e do glutationo redutase durante o desenvolvimento (estado fetal, amamentação, sexo e idade), e propôs o ciclo generalizado de oxidação-redução do glutationo [8].

Estes trabalhos, e em particular o ciclo de oxidação-redução do glutationo, tiveram um enorme impacto na comunidade científica sendo hoje considerados trabalhos clássicos na área dos processos oxidativos em biologia. Este impacto poderá ser avaliado através de vários indicadores, quer quantitativos, quer qualitativos. Os trabalhos realizados por Ruy E. Pinto ao longo da década de 60 foram citados com muita frequência por investigadores de diferentes países, e publicados em várias línguas, incluindo o japonês e o russo. O seu artigo onde se descreve o ciclo de oxidação-redução do glutationo foi citado 225 vezes até à data ${ }^{1}$.

Esquema 1 O esquema original de Pinto e Bartley publicado em 1969

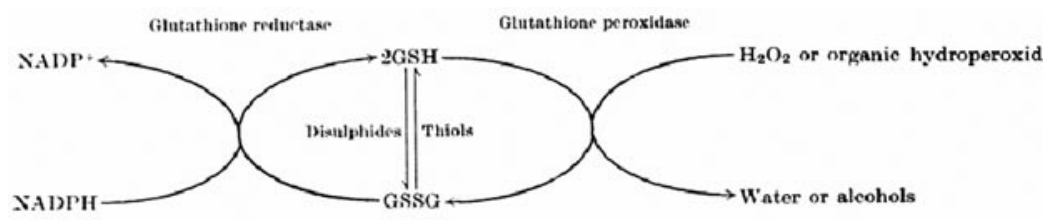

O impacto destas citações no desenvolvimento da área é acrescido, pois estes estudos foram realizados numa fase inicial, como podemos observar por análise da Figura 2.

Os testemunhos pessoais a seguir apresentados são ilustrativos da importância que a contribuição de Ruy Pinto teve na história da bioquímica.

John Rotruck, o autor da descoberta do glutationo peroxidase como sendo um enzima dependente do selénio, um dos trabalhos fundamentais da história da nutrição [9], a propósito do seu artigo publicado na revista Science, em 1973, [10] dá o seguinte testemunho [11]:

Being an enthusiastic graduate student, I read the literature in these areas, especially that on glutathione biochemistry. One of these papers, by R.E. Pinto and W. Bartley [12], was on the effect of age and sex on glutathione peroxidase and glutathione reductase. This paper presented a pathway of g/utathione metabolism that connected glutathione with the enzyme glutathione peroxidase and led me to the hypothesis that selenium could exert "antioxidant activity" as part of glutathione peroxidase.

Sobre o mesmo assunto Richard Ahrens escreve:

A figure in this paper [12] illustrated the proposed relationship of glutathione, glutathione reductase, glutathione peroxidase and NADPH. It was also noted that glucose metabolism is the main source of NADPH. With this figure in front of him, John began to develop that same afternoon a hypothesis that selenium played a role in glutathione peroxidase.

José Viña, tal como Ruy Pinto um discípulo de Sir Hans Krebs desde há muito dedicado à área do envelhecimento, num dos seus artigos, comenta [13]: "Since the pioneering work of Pinto and Bartley (1969) in the sixties, many authors have paid attention to the relationship between glutathione metabolism and aging."

O carácter inovador dos estudos de Pinto na década de sessenta não pode deixar de ser salientado. A existência do próprio glutationo peroxidase era questionada por uma boa parte da co- 
munidade científica da altura. A existência do enzima só viria a ser aceite em 1973 com o já mencionado trabalho de John Rotruck e com a cristalização e confirmação do selénio como co-factor do enzima por Leopold Flohé, em 1973 [14]. No congresso onde Flohé mostrou pela primeira vez fotografias de cristais do glutationo peroxidase, várias comunicações questionavam a existência do enzima [15]. Como acontece com muitos estudos pioneiros, Ruy E. Pinto teve que ultrapassar várias dificuldades técnicas. A inexistência do glutationo oxidado comercial obrigou a sintetizar este composto. Os fracos métodos correntes existentes na época para doseamento do glutationo conduziram ao desenvolvimento de um método potenciométrico utilizando um eléctrodo de mercúrio- glutationo [16]. Mais tarde, Ruy E. Pinto desenvolveu um método enzimático específico para dosear o glutationo oxidado e para determinar as actividades do glutationo peroxidase e do glutationo redutase. Estes métodos continuam ainda hoje a ser utilizados. Desenvolveu ainda uma dieta sintética para estudo de fenómenos de amamentação, não disponivel comercialmente. Mais tarde investigou o efeito da hepatomização e do jejum sobre os dois enzimas [17].

Embora ao longo da carreira de investigação, Ruy E. Pinto tenha trabalhado em vários domínios [18] ${ }^{3}$, nunca deixou esta área, acompanhando o seu desenvolvimento. Assim, após a descoberta de que o glutationo peroxidase era uma seleno-proteína dedicou-se à bioquímica do selénio, para além da do enxofre e do oxigénio que já existia [19]. Continuou também a investigação do metabolismo do glutationo no fígado hepatomizado de rato [20]. Em 1992, Ruy Pinto fundou, juntamente com o então seu aluno de Doutoramento Armindo Salvador, o Grupo de Bioquímica e Biologia Teóricas no IRC. Sob sua supervisão, este grupo desenvolveu trabalho na área dos processos oxidativos em biologia e bioquímica teórica, tendo ao longo de uma década publicado 26 publicações, envolvendo 32 autores de 17 instituições. Os trabalhos realizados foram citados, até à data, 546 vezes (índice h de 14), por 1454 autores $^{4}$. O impacto do grupo junto às fontes de financiamento foi no entanto menos significativo, tendo visto todos os seus projectos recusados. Como consequência, a partir de 2000 a actividade do grupo diminuiu vindo a desintegrar-se em 2003.

Apesar disso, Ruy E. Pinto continuou a exercer uma influência científica nas novas gerações, nomeadamente, através da sua acção pedagógica e na formação de jovens cientistas.

\section{Ruy E. Pinto na Faculdade de Ciências da Universidade de Lisboa}

Em 1971, o Ministério da Educação Nacional regulamentou por decreto [21] a reestruturação das licenciaturas e bacharelatos professados nas faculdades de ciências, na tentativa de procurar modernizar aquelas instituições, criando duas áreas de especialização, uma científica e outra educacional, nos cursos nelas ministrados. Kurt Jacobsohn defendeu a ideia de que a disciplina de bioquímica deveria ser introduzida nos curricula das licenciaturas oferecidas por aquelas faculdades, chegando mesmo a sugerir a criação de uma licenciatura [22]. Em consequência desta posição, a Licenciatura em Química da Faculdade de Ciências da Universidade de Lisboa passou a oferecer a possibilidade de especialização no ramo de química orgânica-bioquímica, mediante um conjunto de disciplinas de opção leccionadas nos $3 .^{\circ}$ e $4 .^{\circ}$ anos, que englobavam as cadeiras de Bioquímica Geral, Bioquímica Complementar e Técnicas de Bioquímica, possibilitando aos alunos a realização de estágios científicos em domínios como a enzimologia, a química de lípidos e a análise bioquímica. Nesta altura, era ainda leccionada a disciplina de Química Médica para os alunos da Faculdade de Medicina da Universidade de Lisboa, e as disciplinas de Bioquímica I e Bioquímica II, da licenciatura em Biologia [23]. Os docentes mais directamente envolvidos na leccionação destas disciplinas, para além de Kurt Jacobsohn, eram Ruy E. Pinto e Renato Leal.

Os planos definidos pelo Ministério, em 1971, foram acompanhados por várias comissões científicas inter-universitá- rias, nomeadas para o efeito, e sofreram várias alterações até 1980 [24]. Na sequência do processo de reformulação dos cursos da responsabilidade das universidades, foi criada a primeira Licenciatura em Bioquímica no país, na Faculdade de Ciências e Tecnologia da Universidade de Coimbra, em 1980 [25], que começou a funcionar no ano seguinte. Seguiu-se a Universidade de Lisboa e a Universidade do Porto em 1981 [26]. A Universidade do Porto propôs ao Ministério da Educação a criação de uma licenciatura conjunta entre a Faculdade de Ciências e o Instituto de Ciências Biomédicas de Abel Salazar ainda nesse ano. Sob proposta da Faculdade de Ciências da Universidade de Lisboa, a licenciatura criada em 1981, viria a ser iniciada em 1982 [27].

Pela formação que Ruy E. Pinto adquirira na Grã-Bretanha e pelas afinidades que o ligavam à tradição britânica nesta área, a Licenciatura em Bioquímica, cuja estruturação liderou, é diferente das suas congéneres no país. Tanto a Faculdade de Ciências de Coimbra como a do Porto apostaram num curso de bioquímica, alicerçado em duas disciplinas, a química e a biologia. ${ }^{5}$

A FCUL tentou afirmar a sua licenciatura dando-Ihe características diferentes. Arquitectada por um grupo coordenado por Ruy E. Pinto, era eminentemente experimental, com várias disciplinas de opção, cobrindo diversas áreas do conhecimento bioquímico e com um estágio de investigação obrigatório. ${ }^{6} \mathrm{Um}$ ramo teórico constituído pelas áreas da bioquímica quântica e da simulação em bioquímica foi uma outra característica pioneira desta licenciatura, tendo cerca de 20 anos de avanço sobre a popularização da bioinformática e a biologia de sistemas.

No entanto, foram necessários alguns anos de negociação com o Ministério da Educação para que este desse o seu aval a uma licenciatura diferente das existentes e que os proponentes argumentavam ser equivalente às do resto da Europa, e promotora de uma interligação às faculdades que incluíam, com certo destaque, o ensino da disciplina as faculdades de Medicina, Veterinária, 
Agronomia, e de Farmácia. Pretendia-se, assim, participar na contínua actualização e aplicabilidade do curriculum de bioquímica a essas licenciaturas, dando aos estudantes da Licenciatura em Bioquímica, uma visão alargada das diferentes vertentes da disciplina [27].

Além disso, pretendia-se também que a licenciatura não fosse o somatório da química e da biologia, como pretendia o Ministério, mas que fosse o resultado duma vivência cultural que apostasse na interdisciplinaridade, reconhecendo como áreas fundamentais, a química, a física e a matemática, sendo a biologia, a informática e a estatística, áreas complementares. A colocação da biologia como disciplina complementar corresponde, claramente, a uma visão fisicista da bioquímica que se traduziu, institucionalmente, no controlo da licenciatura pelo Departamento de Química da FCUL.

Este Departamento assegurou o controlo da licenciatura em dois momentos essenciais do processo de criação da mesma: primeiro, adiantando-se ao Departamento de Biologia ao tomar a iniciativa da criação da licenciatura; segundo, ao incluir na sua proposta a física e a matemática como áreas nucleares da licenciatura, garantiu para a sua proposta os votos favoráveis dos Departamentos de Matemática e de Física. Inicialmente, propunha-se que a licenciatura tivesse quatro anos de escolaridade seguidos de um ano de estágio de investigação científica. No entanto, para obter a aprovação do Ministério da Educação, a licenciatura passou a ser, na sua totalidade, de quatro anos, constituindo o estágio de investigação uma das disciplinas do último semestre do curso [28]. Esta situação resultou, sobretudo, em prejuízo dos primeiros licenciados pois na prática nenhum deles concluiu a licenciatura em menos de cinco anos. ${ }^{7}$

O resultado não foi o desejável mas o possível. Apesar da existência de uma Licenciatura em Bioquímica, em 1997, a profissão de bioquímico ainda não era reconhecida em Portugal, a avaliar pelas palavras de Ruy E. Pinto [1]. "Em Inglaterra eu dizia que era bioquímico; aqui, quando digo que sou bioquímico, toda a gente olha para mim e pergunta-me " mas, é médico? Então é farmacêutico?"... Nós não temos profissão."

A propósito destas palavras, convirá levantar a questão da importância para a afirmação de qualquer área disciplinar da existência de uma sociedade científica activa que socialmente a represente e, ligada a ela, uma publicação especializada que canalize a produção científica. Geralmente, estas etapas são, não só essenciais à consolidação de uma área disciplinar, como à afirmação da identidade profissional dos seus praticantes.

\section{Conclusão}

Ruy E. Pinto é uma figura incontornável da história da bioquímica portuguesa no século XX. Destacou-se no campo científico no âmbito do estudo dos processos oxidativos em Biologia, tornando-se uma referência histórica nesta área de investigação. Por consequência fundou um pequeno grupo de investigação em bioquímica teórica, procurando compreender por simulação, o seu principal objectivo científico: a compreensão dos processos oxidativos biológicos.

No plano institucional foi também um dos principais impulsionadores da institucionalização da bioquímica na Universidade de Lisboa, movimentando-se no sentido da criação de uma licenciatura com características muito próprias, filiadas no exemplo das escolas britânicas.

No plano pedagógico, Ruy Pinto acompanhou não só a preparação dos programas disciplinares de grande parte das disciplinas do curso de Bioquímica na Universidade de Lisboa, como também foi o responsável pela leccionação de algumas delas.

Em qualquer dos percursos que caracterizam a vida e obra de Ruy Pinto é notória a influência da bioquímica britânica. Tendo privado com Hans Krebs, Ruy Pinto tornou-se um dos seus principais seguidores e admiradores. Não admira pois que o tivesse usado como modelo na bioquímica portuguesa, na qual deixa também seguidores.
Notas

1 Pesquisa efectuada no dia 2007/02/27 na base de dados ISI Web of Science.

2 O número de trabalhos na área do glutationo foi obtido fazendo uma pesquisa na base de dados ISI Web of Knowledge usando a palavra "glutathione" restringida ao campo de pesquisa do título. Esta restrição leva a uma sub-estimação do número de artigos mas revelou-se necessária para mostrar a evolução do número de artigos ao longo do tempo. A pesquisa da palavra nos resumos dos artigos levaria a um número mais exacto do número de trabalhos na área, mas dado que nos artigos mais antigos o resumo não se encontra disponível tal pesquisa iria falsear a evolução da curva. As citações dos trabalhos de Ruy E. Pinto referem-se apenas aos seus trabalhos da década de 60 .

3 As outras áreas estudadas, além deste núcleo central de investigação, surgiram na sequência de pedidos para resolução de problemas, nos organismos onde Ruy E. Pinto trabalhava. A título de exemplo podemos citar o estudo clínico-laboratorial dum antidiabético oral, a necessidade de resolver um conflito pernicioso para a economia de Portugal, ou ainda, ao estudo dos alcalóides da Chelidonium Majus L., existente nos Açores.

4 Pesquisa efectuada no dia 2007/02/27 na base de dados ISI Web of Science no campo morada por "BIOQUIM \& BIOL TEOR GRP OR Grp Bioquim \& Biol Teor”

5 Para um conhecimento mais pormenorizado consulte-se os planos das licenciaturas aprovados pelo Ministério da Educação, entre 1979 e 1982, ou os relatórios de avaliação das licenciaturas publicados em 1996, pelo Conselho de Reitores das Universidades Portuguesas.

6 Na impossibilidade de recolhermos informações escritas sobre as diferentes fases do processo de candidatura da Licenciatura em Bioquímica da Universidade de Lisboa, baseámo-nos em depoimentos orais das pessoas que estiveram mais directamente envolvidas no processo, Ruy E. Pinto, Ana Maria Ponces Freire e Jacques Callazans.

7 O entrave à formação experimental especializada ainda presente na década de 80 do século passado parece não ser apenas apanágio da Faculdade de Ciências da Universidade de Lisboa, mas de uma mentalidade de há muito instalada na universidade portuguesa. Seriam necessários mais de 10 anos para que se reconhecesse uma licenciatura com 5 anos, que incluiria o estágio científico no último ano. 
Referências

[1] M. Castanho, "Professor Ruy Pinto, O Rasto de uma Vida dedicada à Ciência - Dureza de Trabalho e Paixão", Boletim da Sociedade Portuguesa de Química 65(1997) 28-37.

[2] H. A. Krebs, (Carta dirigida à Fundação Calouste Gulbenkian datada de 30 de Agosto de 1957).

[3] R. E. Pinto, Curriculum vitae, 1994, 1996, 2001.

[4] R. E. Pinto, "Comparison of the Anaerobic Reduction of Oxidized Gluthathione in Liver Homogenates", Biochemical Journal 79(1961) 43-51.

[5] P. C. Jocelyn, "The Effect of Glutathione on Protein Sulphydryl Groups in Rat Liver Homogenates", Biochemical Journal 85(1962) 480-485

[6] P. C. Jocelyn, "Oxidation of Glutathione and other Thiols by Xanthine Oxidase and Hypoxantine of Rat Liver Homogenates", Nature 202(1964) 1115.

[7] B. O. Christophersen, "Oxidation of Reduced Glutathione by Subcellular Fractions of Rat Liver", Biochemical Journal 100(1966) 95-100.

[8] R. E. Pinto, W. Bartley, "Changes in Glutathione Reductase and Glutathione Peroxidase Activities in Rat Liver Related to Age and Sex", Biochemical Journal (separata) 109 (1968) 1-34; R. E. Pinto, W. Bartley, "The Effect of Age and Sex on Glutathione Reductase and Glutathione Oxidation in Rat Liver Homogenates," Biochemical Journal 112 (1969) 109-115; R. E. Pinto, W. Bartley, "The Nature of the Sex-linked Differences in Glutathione-Peroxidase Activity and Aerobic Oxidation of Glutathion in Male and Female Rat Liver", Biochemical Journal 115 (1969) 449456; R. E. Pinto, W. Bartley, "A Negative Correlation Between Oxygen Uptake and Glutathione Oxidation in Rat Liver", Biochemical Journal 114 (1969) 5-9.

[9] K. J. Carpenter, A.E. Harper, R.E. Olson, "Experiments that changed nutritional thinking", Journal of Nutrition 12 (1997)1017S-1053S.

[10] J.T. Rotruck, A.L. Pope, H.E Ganther, A.B. Swanson, D.G Hafeman, W.G. Hoekstra, "Selenium: biochemical role as a component of glutathione peroxidase", Science 179(1973) 588-590.
[11] J.T Rotruck, "This week's citation classic", Currents Contents 31 (\#29) (1988), 15.

[12] R. E. Pinto, W. Bartley, "The Effect of Age and Sex on Glutathione Reductase and Glutathione Oxidation in Rat Liver Homogenates," Biochemical Journal 112 (1969) 109-115.

[13] J. Viña, J. Sastre, A.L Bruseghini, A Esteras, M. Asensi, "Effect of aging on glutathione metabolism. Protection by antioxidants" in I. Emerit, B. Chance (eds.) Free Radicals in Aging, Birkhauser Verlag, Basel (1992) 136-144.

[14] L. Flohé, W.A Gunzler, H.H. Schock, "Glutathione peroxidase: a selenoenzyme", FEBS Letters 32 (1973) 132-134.

[15] R.E. Pinto, Comunicação pessoal.

[16] R. E. Pinto, "Comparison of the Aerobic and Anaerobic Reduction of Oxidized Glutathione in Liver Homogenates", Biochemical Journal 79 (1961) 43-51; R. E. Pinto, "Alguns Aspectos das Titulações Potenciométricas de Tióis com Eléctrodo de Mercúrio-Tiol", Gazeta de Física 4 (1963) 183-189.

[17] R. E. Pinto, W. Bartley, "Glutathione Reductase and Glutathione Peroxidase Activities in Hepatomous Livers of Rats Treated with Dietylnitrosamine," FEBS Letters 32 (1973) 307-309.

[18] E. Pães, F. J.Silva-Gomes, R. E. Pinto, M.A. Pinto-Albuquerque, H. Farinha, "Ensaio Clínico dum novo Anti-diabético Oral. Glipizida", Revista Ibérica de Endocrinologia 21 (1974) 459-480; J. S. Lopes, R. E. Pinto, M. E. Almendra, J. A. Machado, "Variation of $14 \mathrm{C}$ Activity in Portuguese Vines from 1940 to 1974", Proceeding of International Conference on Low-Radioactivity Measurements and Aplications, Bratislava (1957) 265-268; M. L. Pavão, R. E. Pinto, "Densitometric Assay for the Evaluation of Water Soluble Alkaloids from Chelidonium Majus $L$ in Azores Along One Year Cycle," Arquipélago (separata) (1995) 85-91.

[19] A. Salvador, F. Antunes, R. E. Pinto, "Kinetic Modelling of in vitro Lipid Peroxidation Experiments - "Low Level" Validation of a Model of in vivo Lipid Peroxidation", Free Radical Biology \& Medicine 23 (1995) 151-172; F. Antunes, A. Salvador, H. S. Marinho, R. Alves, R. E. Pinto, “Lipid Peroxidation in Mithocondrial Inner Membranes. I- An Integrative Kinetic Model", Free Radical Biology \& Medicine 21 (1996)
917-943; H. S. Marinho, F. Antunes, R. E. Pinto," Role of Gluthatione Peroxidase and Phospholipid Hydroperoxide Gluthatione Peroxidase in the Reduction of Lysophospholipid Hydroperoxides", Free Radical Biology \& Medicine 22 (1997) 871-883; A. M. Crespo, J. Neve, R. E. Pinto, "Plasma and Liver Selenium Levels in the Rat Supplementation with 0.5, 2, 6 and 15 ppm Selenium in Drinking Water", Biological Trace Element Research 38 (1993) 139-147.

[20] R. E. Pinto, M. F. Duarte, M. L. RodaSantos, V. H. Rosa, " Peroxidation in Hepatomous Liver of Rats Treated with Diethylnotrosamine", Biochemical Society Transactions 8 (1980) 563-564; H. S. Marinho, M. Baptista, R. E. Pinto, "Glutathione Metabolism in Hepatomous Liver of Rats Treated with DietyInitrosamine", Biochimica \& Biophysica Acta 1360 (1997) 157-168.

[21] Decreto n. ${ }^{\circ} 443 / 71$, Diário do Governo, Ministério da Educação Nacional, Direcção Geral do Ensino Superior e das Belas Artes, 23/10/1971.

[22] J. A. Feijó, O Instituto e o Exterior: Relações e Influências, (trabalho não publicado existente no IRC).

[23] Dados fornecidos pelo Departamento de Química da FCUL.

[24] Despacho n. 323/80, Diário da República, Ministério da Educação e das Universidades, 6/10/1980.

[25] Decreto n. 87/80, Diário da República, Ministério da Educação e Ciência, 20/9/1980.

[26] Decreto n. 130/81, Diário da República, Ministérioda Educação e das Universidades, 21/10/1981.

[27] Decreto n. ${ }^{\circ}$ 125/82, Diário da República Ministérioda Educação e das Universidades, 3/11/1982.

[28] R. E Pinto, I. Amaral, "Origens, Desenvolvimento e Panorama Actual da Bioquímica da Faculdade de Ciências da Universidade de Lisboa", Boletim da Sociedade de Química 69 (1998) 23-29. 